\title{
Del condominio a la dicotomía. Las relaciones entre los gobiernos del PT en Brasil con el empresariado internacionalizado brasileño (2003-2016)
}

\author{
Esteban Actis \\ CONICET, Universidad Nacional de Rosario, Rosario, Argentina. \\ Email: e.actis@conicet.gov.ar
}

Resumen:El presente artículo tiene como objetivo comprender la relación entre los gobiernos de Brasil (2003-2016) bajo la conducción del Partido de los Trabajadores (PT) y el gran empresariado brasileño internacionalizado. A partir de la conceptualización ofrecida porGeoffrey Underhill, el trabajo se proponedescribir y analizar los motivos que posibilitaron la conformación de un "Condominio" (2003-2011) así como los factores que explican su deterioro (2011-2014) y su posterior fractura y eclosión (2015-2016). condominio.

Palabras claves: Brasil, partido de los trabajadores, internacionalización,

\section{From condominium to dichotomy. Relations between the Governments of the PT in Brazil andbrazilian multinational corporations (2003-2016)}

\begin{abstract}
This article aims to understand the relationship between the governments of Brazil under the leadership of the Workers' Party (PT) (20032016) and the main internationalized Brazilian corporations. Based on the conceptualization offered by Geoffrey Underhill, the paper intends to describe and analyze the reasons that enabled the formation of a "Condominium" (2003-2011) as well as the factors that explain its deterioration (2011-2014) and its subsequent fracture (2015-2016).
\end{abstract}

Keywords: Brazil, workers’ party, internationalization, condominium

\section{Do condomínio à dicotomia. As relações entre os governos do PT no Brasil e os empresários brasileiros internacionalizados (2003-2016)}

Resumo: O presente artigo pretende compreender a relação entre os governos do Brasil (2003-2016) sob a liderança do Partido dos Trabalhadores (PT) e do grande setor de negócios brasileiro internacionalizado. Com base na conceptualização oferecida por Geoffrey Underhill, o artigo pretende descrever e analisar os motivos 
que permitiram a formação de um “Condomínio” (2003-2011). bem como os fatores que explicam sua deterioração (2011-2014) e sua subsequente fratura e eclosão (2015-2016). condomínio.

Palavras-chave: Brasil, partido dos trabalhadores, internacionalização,

\section{Introducción}

Históricamente el debate político, ideológico y económico en América Latina en torno a la relación entre el Estado y el mercado se estructuró y giró en términos de primacía y dicotomía, dándose en la práctica un juego de suma cero. En los momentos de hegemonía de gobiernos liberales,ha existido una visión de preeminencia del mercado en detrimento de las regulaciones estatales con el objetivo de favorecer la circulación del capital y la ganancia empresarial, provocando en la práctica, la pérdida de cierta autonomía de las capacidades estatales.En otras coyunturas, donde la primacía política estuvo en manos de gobiernos de izquierda/progresistas se concibió- con el fin de evitar constreñimientos- que el poderío del Estadoera inversamente proporcional de los distintos actores del mercado,

Sin embargo, la primacía de una visión antagónica en relación al vínculo estado-mercado, lo que Geoffrey Underhill (2003) define comoStateMarket Dichotomy, no implicó que en muchas experiencias históricas dicho vínculo haya adquirido otra dinámica, en donde la desconfianza y la confrontación fue reemplazada por la colaboración (State-Market Condominium) en el marco de la identificación de intereses comunes y solapados.

Así, en coyunturas históricas particulares, distintos gobiernos con distintas inclinaciones políticas/ideológicas fueron proclives a estar imbricados con ciertos actores socioeconómicos no gubernamentales como por ejemplo los grupos empresariales.Como se corrobora en aquellos países capitalistas exitosos (Chang, 2002) en diversas oportunidades las instituciones y las políticas estatales fueron centrales en los procesos de transformación económica a nivel global y para la propia competencia entre los agentes del mercado. A su vez, robustos mercados han representado para los Estados los medios indispensables de acceso a los procesos de formación y acumulación de riquezas y poder en el orden mundial (Létourneau, 1997, p. 47). En la idea de un condominiosubyace una lógica de ganancias mutuas (win-win game). beneficios económicos para los actores del mercado y beneficios económicos/políticos para los actores gubernamentales.

En este marco, a comienzos del siglo XXI, en el seno del denominado "giro a la izquierda” de América Latina (Moreira, Raus, y Gómez Leyton, 2008) existió la conformación de un fuerte y novedoso “condominio”. En la República Federativa de Brasil, un nuevo gobierno conformado por un par- 
tido de tradición progresista, sindical y obrera -el Partido de los Trabajadores (PT)- bajo el liderazgo de "Lula” Da Silva (2003-2010)y un conjunto de grandes empresas brasileñas internacionalizadas ${ }^{1}$ conformaron una novedosa alianza sustentada en intereses solapados. Sin embargo, durante el transcurso de la primera administración Dilma Rousseff (2011-2014) se comenzó a evidenciar una menor intensidad del condominio como consecuencia de la retracción de las variables que explicaron su conformación (estabilidad económica y la política exterior). En ese sentido, durante el segundo -y corto- mandato de Rousseff (2015-2016) a la luz de la fuerte crisis política/económica y de las investigaciones en torno a la corrupción que salpicó a la clase política y empresarial se produjo el resquebrajamiento del condominio, pasando a una clara situación de dicotomía entre el gobierno del PT y el conjunto de empresas brasileñas internacionalizadas.

A partir de lo dicho, el objetivo del presente trabajo radica en analizar y explicar las razones y motivos que posibilitaron la conformación temporal del "Condominio"así como las circunstancias que explican su posterior debilitamiento y eclosión. El artículo se estructura en tres apartados que analizan los tres momentos señalados, para finalizar con unas conclusiones sobre los resultados obtenidos.

\section{Los gobiernos de “Lula” Da Silva: conformación y auge del condominio entre el gobierno brasileño con el empresariado internacionalizado}

A diferencia de otros casos del denominado "giro a la izquierda" de América Latina donde predominó una fuerte ruptura con el modelo neoliberal -como han sido las experiencias de Venezuela, Ecuador y Bolivia- en el caso de Brasil la implementación de importantes cambios políticos y sociales no implicó un abandono de algunas premisas económicas ortodoxas.

Antes de asumir como presidente el propio Lula Da Silva dio señales de que su gobierno no iba a tener un componente revisionista en materia macroeconómica. El 22 de Junio de 2002 se publicó una carta del electo líder sindical llamada Carta ao Povo Brasileiro, la cual destacaba los compromisos con la estabilidad económica, la responsabilidad fiscal y el respeto de los contratos firmados con los acreedores internacionales.

Desde el año 2003, la flamante coalición de gobierno² liderada por el $\mathrm{PT}^{3}$ implementó una estrategia "híbrida" de desarrollo, combinando aspectos tanto de las corrientes desarrollistas/estructuralistas como de los enfoques monetaristas (Cornel Ban, 2013; Barbosa \& Pereira de Souza, 2010). En la práctica, y a pesar de un fuerte discurso revisionista, hubo una aceptación de ciertas premisas del capitalismo y de la econo- 
mía de mercado que otrora habían sido cuestionadas por el partido. Hacia finales de los años ochenta, las ideas propensas a una ruptura radical con el sistema y el orden vigente fueron perdiendo espacio. El PT transitó, como muchos partidos de la izquierda europea ${ }^{4}$, de una etapa revolucionaria y contraria al reformismo socialdemócrata hacia posturas menos radicalizadas y de aceptación del orden capitalista. A partir de entonces, y durante los años noventa, se produjeron cambios importantes en el seno del PT, particularmente en algunas posturas neurálgicas al interior del partido, que una vez en el poder, se cristalizaron en el seno del Estado brasileño.

Esta mutación en los lineamientos partidarios quedó en evidencia en el ámbito de la política económica de los gobiernos petistas. Souza Ramos (2012, p. 77) señala que una de las principales transformaciones entre las posturas primigenias y las posteriores acciones de gobierno fue el paso de una visión tendiente a "estatizar las propiedades agrícolas a otra caracterizada por incentivar el agrobusiness". En la primera década del siglo XXI aparecen en Brasil intereses domésticos con potencial de expansión internacional los cuales comienzan a demandar políticas públicas que estén acorde a estos nuevos intereses "ofensivos” (Motta Veiga \& Polonia Rios, 2008).Junto al agrobusiness, otro actor nacional que para fines del siglo XX evidenciaba posibilidades de expansión a nivel internacional era un importante conjunto del empresariado nacional. Motivo por el cual, siguiendo con la comparación que nos propone Souza Ramos, la postura del PT con respecto a las empresas multinacionales puede ser resumida como el paso de una visión proclive a nacionalizar las empresas extranjeras y combatir al capital trasnacionalaotra caracterizada por el apoyo a la expansión de las empresas multinacionales brasileñas.

Al contrario de lo que uno imagina para un gobierno de izquierda, desde el Palacio del Planalto, se asoció el "interés nacional" con el interés privado de las "grandes empresas". Las principales firmas brasileñas con importantes inversiones en el exterior fueron colocadas por el gobierno como los "motores del desarrollo nacional, símbolo de un Brasil moderno y capaces de competir en el mercado internacional contra las grandes empresas extranjeras" (Garcia, 2011, p. 23). Los intereses particulares de actores específicos del mercado fueron representados como universales para toda la nación ${ }^{5}$.

Desde esta perspectiva, retomando lo señalado en la introducción, la relación del PT con el mercado, especialmente con la gran burguesía nacional internacionalizada, viró de una lógica de State-Market Dichotomy a unamodalidad de condominio. Ahora bien, ¿qué hechos y acontecimientos nos llevan a señalar la emergencia de una etapa signada por un government-business collaboration, entre los gobiernos de Lula Da Silva con el capital brasileño internacionalizado?

En primer lugar,la decisión del presidente Lula de nombrar como 
su Vice-presidente al empresario José Alencar, fundador de una de las mayores empresas textiles de Brasil -Coteminas- fue un claro mensaje pro-mercado de un gobierno que generaba todo tipo de dudas sobre su orientación económica. Con Alencar, el establismentproductivo consiguió llegada directa al gobierno. La alianza política de Lula con Alencar -a través de él gran parte de la burguesía brasileña- fue uno de los elementos centrales que posibilitó el triunfo del Lula Da Silva en las elecciones del 2002. En palabras del propio ex presidente:

\begin{abstract}
“(...) precisaba encontrar un vice-presidente que no fuera del PT y que no fuese más de izquierda que yo, tendría que ser un poco más conservador y una persona que representase otros segmentos de la sociedad. Un día fui invitado para visitar a un empresario que estaba conmemorando los 50 años de vida empresarial en el estado de Minas Gerais [...] fui convencido por mis compañeros de ir al aniversario del entonces senador, presidente de Coteminas, José Alencar. Nunca había visto a José Alencar, nunca había tenido una conversación con él. Después de que terminó su discurso contando su historia, le dije a mis compañeros: acabé de encontrar al vice-presidente de la República que necesito para conquistar los votos que preciso obtener. Ya estaba cansado de tener el 30\% de los votos, precisaba tener el 50\% y, ciertamente, José Alencar dio una mano extraordinaria ${ }^{6}$ ”.
\end{abstract}

Por su parte, Luiz Fernando Furlan fue otroempresario que formó parte del gabinete del Presidente. Furlan, quien fuera presidente del Consejode Administración de la empresa Sadia entre 1993 y 2003, desarrolló un rol clave en la política de comercio exterior en el primer mandato de Lulacomo Ministro entre 2003 y 2007, del Ministerio do Desenvolvimento, Indústria e Comércio Exterior.Una vez que dejó el gobierno, regresó a la conducción de la empresa Sadia en un contexto de fuerte crisis de la firma alimenticia que finalizó con la fusión con su principal competidor (Perdigão) para crear, en 2009, el grupo Brasil Foods (BRF). Asimismo, las estrechas relaciones interpersonales del presidente Lula Da Silva con Emilio y Marcelo Odebrecht (Vigna, 2013). ambos presidentes del homónimos holding, evidenciaron la cercanía que existió entre una de las mayores empresas mundiales del rubro ingeniería y construcción (empreiteras) con el gobierno petista.

Otro dato que demuestra el acompañamiento del empresariado internacionalizado al modelo político y económico que implementó el PT, está relacionado con las donaciones que efectuaron las firmas en las campañas presidenciales del 2006 y 2010. 


\section{Cuadro $N^{\circ} 1$ \\ Donaciones realizadas por las principales empresas internacionalizadas brasileñas a los candidatos presidenciales, elecciones 2006 y 2010, en Reales.}

\begin{tabular}{|c|c|c|c|c|}
\hline Empresa & $\begin{array}{c}\text { Donaciones } \\
\text { para la } \\
\text { campaña Lula } \\
\text { (2006) }\end{array}$ & $\begin{array}{c}\text { Donaciones } \\
\text { para la } \\
\text { campaña } \\
\text { Alckimin } \\
\text { (2006) }\end{array}$ & $\begin{array}{l}\text { Donaciones para } \\
\text { la campaña } \\
\text { Rousseff (2010) }\end{array}$ & $\begin{array}{c}\text { Donaciones para } \\
\text { la campaña } \\
\text { Serra (2010) }\end{array}$ \\
\hline Friboi-JBS & 2.502 .000 & & 12.000 .000 & 6.000 .000 \\
\hline Gerdau & 3.100 .000 & 3.000 .000 & 6.000 .000 & 3.100 .000 \\
\hline Odebrecht & 150.000 & & 2.400 .000 & \\
\hline Marfrig & 200.000 & & & \\
\hline Vale & & & 6.010 .000 & \\
\hline Suzano & 475.000 & 415.000 & 1.000 .000 & 860.104 \\
\hline Camargo Correa & 2.500 .000 & 400.000 & 13.000 .000 & 6.000 .000 \\
\hline Marcopolo & 50.000 & 35.000 & 200.000 & 50.000 \\
\hline WEG & & & 50.000 & 50.000 \\
\hline Votorantim & 1.000 .000 & 356.056 & 2.500 .000 & 1.630 .000 \\
\hline Embraer & 1.300 .000 & 1.000 .000 & 675.000 & 595.000 \\
\hline Andrade Gutierrez & 1.000 .020 & 1.500 .000 & 15.000 .500 & 14.800 .000 \\
\hline Randon & 35.000 & 15.000 & 30.000 & 30.000 \\
\hline Brazil Food & & & 550.000 & 500.000 \\
\hline Total & 12.312 .020 & 6.721 .056 & 59.415 .500 & 33.615 .104 \\
\hline
\end{tabular}

Fuente: elaboración propia en base a Valdez (2011)

Estos últimos datos nos indican la estrecha relación del empresariado con la clase política brasileña ${ }^{7}$ y, en particular, el apoyo (económico y político) a un modelo de gestión gubernamental particular encabezado por un partido de izquierda cuya base social originaria estuvo vinculada a los trabajadores. En este sentido, que una de las mayores fuentes de financiamiento del Partido de gobierno sea el aporte de los grandes grupos económicos privados es una clara señal de las mutaciones sufridas por el PT en los últimos diez años. En términos temporales, si con Fernando Henrique Cardoso las grandes empresas brasileñas se modernizaron y adaptaron a un nuevo entorno económico, con Lula el capital se conglomeró e internacionalizó a escala global (Actis, 2015).

Como bien señala Zibechi (2012). en la primera década del siglo XXI Brasil acentuó, independientemente de la visión progresista de su gobierno, el rol de potencia regional incorporando aspectos típicos de actores con vocación imperial como lo es la fuerte expansión del empresariado nacional en todo su contexto contiguo o "patio trasero".

Como ya mencionamos, la conformación de todo condominio ${ }^{8}$ obedece a la aparición de intereses solapados. Si para los actores del mercado, los beneficios esperados son principalmente de tipo económico, para aquellos que conducen los designios de un Estado, son tan importantes las ganancias en el plano económico como en la arena política. En ese contexto, además del crecimiento y estabilidad económica que consiguió el gobierno 
de Lula Da Silva en sus ochos años de mandato, la otro política pública que cautivo al empresario brasileño fue la política exterior. La búsqueda de un mayor protagonismo internacional de Brasil sustentada en la denominada “diplomacia presidencial” (Cason \& Power, 2009) fue funcional a los intereses de un importante conjunto de empresas brasileñas con perspectiva de iniciar o consolidar sus inversiones en el exterior (Vasconcellos, 2014). Por su parte, la presencia diversificada de las empresas brasileñas por el mundo representó para el gobierno del PT un recurso de poder duro que sustentó y potenció tanto los nuevos vínculos Sur-Sur como los tradicionales lazos con los países en desarrollo (Actis, 2012).

El por entonces Presidente de la firma minera Vale, Roger Agnelli, elogió el modelo de vinculación de Brasil con el mundo, como así también el rol de la "diplomacia presidencial":

"las visitas políticas del presidente abren puertas, trayendo a los empresarios, presentando el potencial de África a los empresarios brasileños. El presidente Lula ha hecho un trabajo maravilloso en esa dirección, él nos ayudó en Mozambique, en Angola, en Zambia, en todos los lugares, él nos presenta, facilita nuestra integración en los países [...] Las relaciones institucionales entre países es fundamental para que las empresas desarrollen negocios"

Por su parte, desde el ejecutivo también se hizo público el convencimiento del rol fundamental que tenían las "multinacionales brasileñas” para la estrategia de inserción internacional de Brasil. Según el ex Canciller Celso Amorim "La creciente presencia de empresas brasileñas en el exterior, que es de manera diversificada, fortalece las líneas generales de la política exterior $^{10}$ ”. Más contundente aún fueron las palabras del propio Presidente Lula Da Silva. En el año 2008, en un discurso en Lisboa afirmó:

"Al final de cuentas una empresa brasileña en el exterior es siempre una bandera brasileña en el exterior, abre un espacio político de negociación, abre un espacio económico de comercialización [...] muchas empresas finalmente se están uniendo al club de las grandes trasnacionales. Son compañías que invierten en los mercados de nuestros socios, complementan el trabajo que viene realizando nuestra diplomacia ayudando a fortalecer y profundizar, con hechos concretos nuestras relaciones" 11

La fortaleza del Condominio analizado hacía suponer que en Brasil se iba en camino a superar la lógica de "ámbitos privilegiados de acumulación” propia del capitalismo latinoamericano (Castelani, 2009). caracterizado por una alianza cortoplacista entre el gran empresariado y el Estado donde un grupo reducido de firmas logran internalizar amplios márgenes de beneficios extraordinarios. Sin embargo, la continuidad del proyecto de gobierno bajo otro liderazgo y la emergencia de otro contexto alteraron los cimientos en los cuales se construyó el Condominio. 


\section{El primer gobierno de Dilma Rousseff (2011-2014): erosión de los pilares que sustentaron el condominio}

Dilma Rousseff asumió la presidencia de Brasil el 1 de enero del 2011. La nueva mandataria se había desempeñado primero como Ministra de Minas y Energía y luegocomo Jefa de Gabinete de su predecesor. La elección de Rousseff significó la esperanza de una continuidad y profundización de los lineamientos centrales del gobierno de Lula Da Silva.

El inició de su mandato, la Presidenta mostraba que la fortaleza del condominio con los grandes empresarios brasileños estaba intacta. En ese marco, no sorprendió que Jorge Gerdau (presidente del Grupo homónimo) presidiera un "Grupo de Gestión de la Competitividad" que vinculaba directamente al empresariado con las más altas esferas delPalacio del Planalto.

Sin embargo, con el transcurrir del tiempo, la fuerte y estratégica alianza que el gobierno de Lula Da Silva había construido con el empresariado internacionalizado comenzó a desgastarse producto de una retracción relativa de los dos pilares que posibilitaron su conformación: el crecimiento/ estabilidad económica y la política exterior. En ambas dimensiones los intereses convergentes comenzarona dar paso a intereses divergentes.

Con respecto al primer aspecto, a partir del año 2011 la economía brasileña sufrió una significativa retracción del crecimiento ${ }^{12}$. Además, la elogiada estabilidad macroeconómica que supo mantener el ex líder sindical, comenzó a tambalear producto de un escenario que requería cada vez más de políticas contracíclicas ${ }^{13}$. Esta cuestión derivó en la emergencia de tensiones producto de algunos desacuerdos entre el gobierno de Dilma Rousseff y los empresarios internacionalizados.El punto más álgido ocurrió en octubre del 2013 cuando el Ministro de Hacienda, Guido Mantega,presentó un proyecto para regular la tributación de los lucros de las corporaciones brasileñas que operan en el exterior. El gobierno apuntó que las empresas debían abonarle al Estado un monto aproximado de 25 mil millones de Reales por "deudas tributarias" como consecuencias de sus actividades internacionales.

Este proyecto generó fuertes críticas por parte de las firmas y las organizaciones empresariales -como fue el caso del Instituto de Estudos para o Desenvolvimento Industrial (IEDI)- las cuales cuestionaron las implicancias negativas del nuevo esquema tributario. Meses más tarde, el ministro se reunió con 18 empresarios quienes le manifestaron que, bajo esas condiciones, el proyecto afectaría la competitividad de las empresas brasileñas debido a que las mismas pagarían más tributos que sus competidoras internacionales. En ese marco, Mantega manifestó la voluntad de generar un "grupo de trabajo" con el objetivo de introducir cambios al proyecto original (Folha do S.Paulo, 2014). El malestar provocado por lainiciativa de reforma tributaria fue señalado por Abilio Diniz, Presidente del Consejo de Administraciones de Brazil Food: “(...) el tema es una 
pulseada con el gobierno, esto demuestra que no siempre estamos de luna de miel, más allá de la amistad que existe con Dilma Rousseff y el ex presidente Lula” (Valor Económico, 2014).

A pesar de las diferencias, el propio Diniz, junto con representantes de Camargo Correa, Odebrecht y Andrade Gutiérrez, se reunieron con la presidenta Rousseff en la apertura del $14^{\circ}$ Congreso de la Federación de Asociaciones de Comercios del Estado de San Pablo (Facesp) en noviembre de 2013. Dicho evento parecía indicar que, más allá de algunas disputas y de un pobre contexto económico, el diálogo fluido entre el gran empresariado y el gobierno seguía vigente.

No obstante, y a pesar del éxito de las presiones realizadas por el empresariado a favor de cambios a la iniciativa original, la decisión política de la administración Rousseff en relación a la ley de tributación implicó un cambio de actitud hacia los grandes empresarios brasileños. En mayo de 2014, la primera mandataria vetó algunos artículos de la Medida Provisória 627/2013sancionada por el Congreso Nacional, dejando sin efecto las cláusulas que eximían a las empresas vinculadas a la construcción e ingeniería de un aumento inmediato de tributación sobre grandes obras en el exterior con fecha anterior a la entrada en vigencia de la Ley.

De forma paralela, la otra política que dejó de ser funcional al mantenimiento del condominio fue la política exterior. Durante la gestión de Rousseff existió un claro declíniorelativo de la inserción internacional de Brasil vis a vis la "era” Lula (Cervo \& Lessa, 2014). Si bien no debe subestimarse la emergencia de un contexto externo menos permisivo, hubo una merma en la intensidad diplomática, principalmente, por la desaparición del canal en el cual se había estructurado la política exterior de Lula Da Silva, como fue "la diplomacia presidencial". El menor activismo presidencial del Rousseff (Malamud, 2011) se tradujo operativamente en un menor involucramiento de la presidenta en el exterior ${ }^{14}$ y por ende en una menor capacidad de traicionar los intereses empresariales. Asimismo, al contrario de lo que ocurrió con Lula Da Silva, en el rastreo de los distintos discursos públicos de Rousseff no se encuentran alusiones a la importancia del rol de la política para la concreción de negocios. En realidad existió un retorno a la idea de que negocios y política corren por distintos andariveles. En ese contexto, la disminución de los viajes presidenciales al exterior sumado a la ausencia de un Ministro de Relaciones Exteriores con alto perfil ${ }^{15}$ y cierta desconfianza de Rousseff sobre la utilidad de la práctica diplomática han llevado a una "retracción estratégica de la política exterior" (Stuenkel, 2014). Un ejemplo de como la política exterior dejó de ser funcional a los intereses del empresariado internacionalizado ${ }^{16}$ se puede observar en el caso de la relación bilateral entre Argentina y Brasil.

En noviembre de 2009, producto de las repercusiones de la crisis económica internacional en ambos lados de la frontera, se recrudecieron las medidas restrictivas en el comercio bilateral. En este marco, al reclamo de los empresarios brasileños por la extensión del plazo de las licencias no 
automáticas a las importaciones aplicadas por el gobierno argentino se sumó la queja argentina por el freno en la frontera brasileña de camionetas y automóviles. En un encuentro entre Lula Da Silva y Cristina Fernández Kirchner en Brasilia además de intentar canalizar los contrapuntos comerciales, los presidentesanunciaronque la empresa brasileña Vale iba a realizar -a partir del 2010- una mega inversión en la provincia de Mendoza para la explotación de potasio estimada en 4.500 millones de dólares. Ante tal situación, el asesor presidencial Marco Aurelio García aprovechó la oportunidad para exponer a las inversiones de la compañía como un ejemplo del interés de Brasil en estimular la integración productiva entre los dos países“(...) que fue apuntada por Lula como la única forma de superar los recurrentes conflictos comerciales entre ambos” (Portal Mercosur-ABC, 2009).

A comienzos del 2013, la empresa brasileña decidió paralizar su inversión en la Argentina. La metamorfosis del contexto económico argentino como el cambio de la estrategia global de la firma fueron determinantes para la decisión final de la empresa. Asimismo, la menor capacidad relativa de la diplomacia del gobierno de Rousseff de alcanzar resultados en la intermediación gubernamental entre el capital brasileño y el gobierno argentino también tuvo un peso determinante a la hora de explicar el abandono de un proyecto que había sido ponderado años atrás. Cabe señalar, que el presidenteLula Da Silva supo, a partir del rol central de la diplomacia presidencial, sobreponer la lógica política a los aspectos técnicos en situaciones sensibles para la Argentina ${ }^{17}$. La capacidad del presidente Lula de hacer prevalecer la carta política no solo obedeció a su carisma individual, sino también, a los márgenes más flexibles que le otorgaba la solidez del condominio con el capital internacionalizado.

Ante la parálisis de la obra y los pocos avances en las negociaciones en los mandos medios de ambas burocracias, Dilma Rousseff arribó a la Argentina en abril del 2013 para un encuentro con la primera mandataria de este país. Al salir del encuentro, Rousseff declaró: “el diálogo es el mejor camino para encontrar soluciones y Vale va a encontrar el camino para construir un acuerdo con las autoridades argentinas”. La temática de la compañía Vale fue el único aspecto bilateral tratado en la reunión que fue mencionado por las presidentes en su declaración conjunta a la prensa (Valor Económico, 2013a).

No obstante, de manera simultánea a las palabras de la Presidenta donde afirmaba que la multinacional iba a buscar un acuerdo, el propio presidente de la firma, Murilo Ferreira -que no formó parte de la comitiva presidencial- declaró que la empresa "no llevaría adelante el proyecto" (Valor Económico, 2013b). Las afirmaciones de Ferreira provocaron un duro golpe al intento de contribuir a la solución por parte del gobierno y una deslegitimación importante de la palabra presidencial. Esta situación conllevó al malestar del gobierno, expresado por el Asesor Presidencial en Asuntos Internacionales, Marco Aurelio García: 


\begin{abstract}
"Vale es una empresa privada, sin embargo, tratamos de ayudar en el acercamiento de posiciones entre Vale y el gobierno argentino. Tengo entendido que había una propuesta importante, de buena calidad, sobre eso y tanto la presidenta Cristina como la presidenta Dilma fueron sorprendidas por una declaración de la retirada de Vale que se hizo a la hora en que las dos estaban reunidas. Eso nos parece un gran error, no la retirada sino hacer una declaración cuando dos presidentes están reunidas” (La Nación, 2013)
\end{abstract}

Otro acontecimiento que tuvo fuerte repercusión al interior del condominiofueron las palabras pronunciadas, en abril del 2014,por Jorge Gerdau en el marco de un seminario en la ciudad de Porto Alegre. En dicha oportunidad el empresario criticó tanto aspectos vinculados a la política económica como a ciertos lineamientos de la política exterior. Según Gerdau: “Los países del grupo del Pacífico vienen creciendo un 5 o 6\% al año y se aproximan al bloque norteamericano que se está integrando con Europa, en cambio nosotros nos quedamos amarrados al Mercosur bolivariano... ¿ ¿será que Cristina Kirchner tiene que decir lo que Brasil debe hacer? ${ }^{18}$ ”

Por último, y estrechamente vinculado, es menester señalar la negativa de dos importantes empresarios para integrar al gobierno de Dilma Rousseff como otro dato importante a la hora de mostrar los desajustes en el condominio. A diferencia de lo que aconteció con Lula Da Silva, tanto Josué Gomes da Silva -hijo del ex vicepresidente José Alencar- como Abilio Diniz -Presidente de la firma Brazil Foods- rechazaron la invitación para asumir el Ministério do Desenvolvimento, Indústria e Comércio Exterior (MDIC) luego de la renuncia de Fernando Pimentel en febrero de 2014.

\title{
El breve segundo mandato de Dilma Rousseff: eclosión del condominio y surgimiento de la dicotomía (2015-2016)
}

El día 1 de enero del 2015, Dilma Rousseff asumió su segundo mandato presidencial luego de imponerse ajustadamente en segunda vuelta al vencer al candidato del PSDB Aecio Neves. A diferencia de las tres elecciones anteriores donde la coalición liderada por el PT se impuso holgadamente, en esta oportunidad se evidenció una polarización política y social muy marcada. Si bien el paulatino deterioro de la situación macroeconómica como así también los ajustes en materia de política exterior generó, como se evidenció, un indudable resquebrajamiento del condominio, el empresariado internacionalizado apostó nuevamente por la continuidad. Por ejemplo, el empresario Abilio Diniz, que como indicamos tuvo importantes contrapuntos con el gobierno de Rousseff, decidió apoyar públicamente la candidatura presidencial de la Presidenta ${ }^{19}$.

La mayor evidencia de que a pesar de la menor intensidad relativa del Condominio bajo la primera presidencia de Rousseff el mismo seguía vigente fueron nuevamente las donaciones realizadas por las principales multina- 
cionales brasileñas en la campaña electoral del año 2014. De acuerdo a las tensiones descriptas supra, podría esperarse un fuerte respaldo del gran capital brasileño a los candidatos de la oposición. No obstante, al igual que en las pasadas contiendas electorales, el empresariado brasileño reforzó la apuesta (de acuerdo a sus donaciones) a una continuidad de Rousseff y de la coalición de gobierno bajo la conducción del PT.

\section{Cuadro $\mathrm{N}^{\circ} 2$ \\ Donaciones realizadas por las principales empresas internacionalizadas brasileñas a candidatos presidenciales, elecciones 2014, en Reales.}

\begin{tabular}{|l|r|r|}
\hline \multicolumn{1}{|c|}{ Empresa } & $\begin{array}{c}\text { Dilma Rousseff } \\
\text { (PT) }\end{array}$ & Aecio Neves (PSDB) \\
\hline JBS & 54.000 .000 & \\
\hline Odebrecht & 3.000 .000 & \\
\hline Gerdau & 5.000 .000 & \\
\hline Camargo Correa & 2.000 .000 & \\
\hline WEG & 150.000 & \\
\hline Embraer & 500.000 & \\
\hline Marcopolo & 50.000 & \\
\hline Andrade Guitierrez & 20.000 .000 & \\
\hline Randon & 30.000 & \\
\hline Brazil Foods (BRF) & 1.500 .000 & \\
\hline OAS & 10.000 .000 & \\
\hline VALE & 2.500 .000 & \\
\hline Braskem & 2.250 .000 & \\
\hline Itaú/Unibanco & 4.000 .000 & 2.000 .000 \\
\hline Votorantim & & \\
\hline Total & $\mathbf{1 0 4 . 9 8 0 . 0 0 0}$ & \\
\hline
\end{tabular}

Fuente: elaboración propia en base a datos del Tribunal Superior Electoral de Brasil.

Disponibles en: http://inter01.tse.jus.br/spceweb.consulta.receitasdespesas2014/

resumoReceitasByComite.action

Sin embargo, desde el inicio del segundo gobierno de Rousseff, Brasil transitó por un período de crisis económica y política que minó poco a poco la legitimidad de la Presidenta. La centralidad de la temática de la “corrupción” en la vida política del gigante sudamericano, el rápido abandono de la agenda prometida de campaña electoral, la aceleración del deterioro económico así como la virulencia de los sectores opositores al gobierno (Tible y Moraes, 2015) dieron como resultado el surgimiento de una profunda crisis al interior de la coalición de gobierno y de los sectores de la sociedad civil que habían apostado por un nuevo gobierno del PT, entre ellos las grandes empresas internacionalizas de Brasil. Si desde el año 2003, el poder conseguido por el PT había funcionado como fuerza centrípeta, atrayendo a sector ajenos a la tradición política del partido como fueron los principales grupos empresariales, para mediados del 2015 el efecto fue in- 
verso, es decir un proceso centrífugo del poder. Desde ese momento, en Brasil comenzó a sobrevolar el fantasma del juicio político (impeachment) dado las fisuras sufridas al interior de su sector aliado, principalmente por el paulatino alejamiento por parte del PMDB (Pereyra Doval y Actis, 2016). La votación en mayo del 2016 de la Cámara de Diputados de Brasil para autorizar el inicio del juicio político apartó provisoriamente a Rousseff (a finales de Agosto fue su destitución definitiva) dando por finalizado más de 13 años de la experiencia del PT en el gobierno de Brasil.

En esa especial coyuntura debe interpretarse la eclosión del Condominio aquí descripto dado la paulatina desaparición de aquellos intereses solapados que lo hicieron posible. El agravamiento de la crisis económica/ política y la temática “corrupción”, en la cual los principales involucrados eran el partido de gobierno y muchas de las empresas multinacionales brasileñas, se transformaron en variables explicativas para comprender la bifurcación de los caminos y la irrupción de una dinámica próxima a lo que Underhill señala como Dicotómica. La visibilidad política del esquema de corrupción mostró que el solapamiento de intereses entre un sector específico del empresariado internacionalizado como son las empresas de ingeniería y construcción (las denominadas empreiteras) con el Estado, tenía en su parte más profunda aspectos ilícitos. Como evidenciaremos a continuación, el nuevo contexto se transformó en un factor restrictivo para la concreción de escenarios de “ganancias mutuas” en el vínculo gobierno del PT- grandes compañías brasileñas internacionalizadas.

El desgaste entre las multinacionales brasileñas aliadas y el gobierno comenzaron apenas iniciado el nuevo mandato Rousseff. La elección de Kátia Abreu como Ministra de Agricultura generó un malestar entre las multinacionales brasileñas del rubro ganadero dado la oposición pública de esta última con respecto a la concentración de las grandes empresas en la explotación y producción pecuaria. A pesar de la oposición de Joesley Batista, CEO de JBS -principal firma donante de la campaña electoral- y de los intentos de muchos allegados por cambiar el perfil seleccionado, Rousseff se mantuvo firme en su decisión. (Folha do S.Pablo, 2014b). No obstante, la intermediación gubernamental para profundizar los negocios en Venezuela, en un contexto de crisis alimentaria del país caribeño ayudó a encauzar los contrapuntos ${ }^{20}$

Durante todo el año 2015, Brasil pasó del estancamiento económico a una fuerte recesión. En el primer año del segundo mandato de Rousseff, la economía cayó un 3,8\% siendo uno de los peores desempeños de los países emergentes. La fuerte caída de la actividad impactó en los planes de negocios de muchas firmas brasileñas internacionalizadas, que como bien señalamos, independientemente de su proyección global, conservan en el mercado nacional una importante porción del plan de negocios. Un ejemplo fue el caso de la fabricante de ómnibus Marcopolo, quien en septiembre de 2015 denunció el incumplimiento del gobierno federal de un crédito por 130 millones de Reales en el marco del Programa “Caminho da Escola”, una iniciativa implementada por los gobiernos petistas. La firma, que concluyó 
el balance de 2015 con una caída de las ingresos netos de un 35\%, indicó que "la actual coyuntura económica y la inestabilidad política de Brasil afectaron el segmento de bienes de capital... también la menor demanda de vehículos escolares provenientes del programa Caminho da Escola como los atrasos en los pagos del gobierno federal afectaron los resultados de los negocios” (Valor Econômico,2016, p. 13).

Las dificultades presupuestarias del gobierno también condicionaron a otra importante firma como Embraer. A mediados del 2015 el Ministerio de Defensa comenzó a retrasar los pagos del programa KC-390 (aviones cargueros) generando un perjuicio de 400 millones de dólares. Por su parte, una decisión de la propia Rousseff en materia de política exterior también afectó los intereses del fabricante de aviones. Luego de la ejecución de dos ciudadanos brasileños en Indonesia, y ante la ineficacia del pedido de clemencia de Rousseff, la mandataria rechazó las credenciales del nuevo embajador indonesio para comenzar a trabajar en Brasil. Esta situación tensó la relación bilateral y perjudicó principalmente a Embraer, empresa con importante presencia en dicho mercado asiático. El vice-presidente de Indonesia, Jusuf Kalla, respondió con la advertencia de la cancelación de un contrato de 150 millones de dólares para la compra de ocho aviones EMB-314 Super Tucano fabricados por la empresa brasileña (Estadão, 2015)

Además de la fragilidad económica, el recrudecimiento de la crisis política que atravesó todo el segundo mandato de la Presidenta Rousseff también se transformó en una variable central a la hora de explicar la ruptura del Condominio. Un caso emblemático en la evolución señalada en el trabajo (auge, debilitamiento y eclosión) es el del holding Gerdau. En un evento organizado por los sindicatos de la industria de la transformación, empresario Jorge Gerdau Johannpeter planteó su "irritación” con el momento que vivía Brasil y expreso la necesidad de "salir a la calle ${ }^{21}$ " como señal de protesta en un claro mensaje opositor al ejecutivo brasileño, en un contexto político donde la "calle" se transformó en el símbolo de la polarización política en Brasil. No cabe duda que la "calle” fue un factor central para explicar la salida anticipada de Rousseff.

En relación a esta variable, otro indicador que refuerza nuestro argumento se vincula con la dinámica que adquirió los contactos entre el gobierno y la multinacional Vale. Al igual que en el caso de Gerdau, también es posible visualizar un proceso de deterioro en la vinculación que eclosiona en esta última etapa. La decisión de Rousseff en marzo de 2015 de elegir a Murilo Ferreira, Presidente de Vale, para que ocupe la Presidencia del Consejo de Administración de Petrobras evidenció que, a pesar de algunos desacuerdos, la alianza seguía siendo fuerte. Además, el incremento de los negocios de la firma en China, bajo la profundización de la relación bilateral de Brasil con el gigante asiático ${ }^{22}$, auguraba para el Palacio del Planalto un firme aliado en la difícil coyuntura política. Sin embargo, con el paso de los meses, el vínculo del gobierno con la firma se fue deteriorando a la luz de pérdida de liderazgo de la primera mandataria. Para agosto Ferreira pidió licencia de su cargo en Petrobras por no estar conforme con el plan de 
negocios de la petrolera. Seguidamente, a principios de noviembre se produjo el rompimiento de una barrera de residuos de la empresa Samarco (controlada por Vale y la anglo-australiana BHP Billiton) en un proyecto en la ciudad de Mariana (Minas Gerais). El desastre ambiental ocasionado fue indicado como el mayor desastre socio-ambiental en la historia de Brasil. La reacción de Rousseff fue ordenar al ministro de la Casa Civil, Jaques Wagner, a que delegue todos los costos de la tragedia a la empresa Samarco y a sus controladoras, entre ellos Vale. Por su parte, Ferreira señaló que Vale no era responsable de lo sucedido no teniendo ninguna responsabilidad ${ }^{23}$. Este hecho es un claro ejemplo de la irrupción de intereses antagónicos entre los actores.

Por último, es dable indicar que la variable “corrupción” fue central a la hora de explicar la ruptura del Condominio entre el empresariado internacionalizado brasileño y el gobierno del PT. En junio de 2014 se conoció públicamente una investigación iniciada en el Estado de Paraná con el fin de desbaratar una organización dedicada el crimen de lavado de dinero (se sospechaba la utilización de una red de gasolineras y lavaderos de autos para mover los recursos ilegales, de ahí el nombre de la operación Lava Jatos). En el avance de la investigación se involucró a funcionarios de la estatal Petrobras y a directivos de empresas contratistas de esta última (Odebrecht, OAS, Andrade Gutierrez, Camargo Correa, Queiroz Galvão, entre otras) principales empresas del rubro de la ingeniería que habían potenciado su accionar desde los gobiernos de Lula (tanto a nivel nacional como internacional de la mano del Estado). y a muchos políticos de distintos partidos políticos, principalmente de la coalición de gobierno liderada por el PT. La acusación del Ministerio Público indicaba que el club de una veintena de constructoras conseguía licitaciones de Petrobras de manera fraudulenta pagando sobornos, los cuales retornaban para financiar la política o directamente iban a los bolsillos de distintos políticos.

Desde mediados de 2015 fueron detenidos los empresarios Marcelo Odebrecht, Otávio Marques de Azevedo (Presidente de Andrade Gutierrez) y Júlio Carmargo (Camargo Correa) y muchos referentes del PT como José Dirceu, Delcídio do Amaral y João Vaccari. A partir de entonces, el vínculo entre muchas de las empresas que habían sido el núcleo del Condominio (el caso más emblemático era el de Odebrecht) con el gobierno entró en un impasse debido a que los intereses eran suma cero, en un intento desesperado por los distintos actores de despegarse de las acusaciones ${ }^{24}$. La investigación del Lava Jato produjo un efecto dominó que contribuyó a deteriorar la situación económica y política, minando la credibilidad de Dilma (Ribeiro et.al., 2016). Si bien es cierto que el crimen de responsabilidad que llevó a Rousseff al juicio político a Rousseff no estaba vinculado con actos de corrupción (viciando su legalidad) la condena social si se centraba en la falta de transparencia del gobierno y del Partido (Peralta, 2016). Por su parte, las investigaciones se profundizaron en torno al vínculo del ex presidente Lula con dichas empresas, como así también el rol del Banco Nacional de Desarrollo (BNDES) en operaciones para que dichas firmas brasileñas concreten licitaciones para obras en el exterior ${ }^{25}$. 


\section{Conclusiones}

En mayo de 2016 se interrumpió 13 años de gobiernos conducido por el Partido de los Trabajadores en Brasil. El segundo mandato constitucional de Dilma Rousseff no concluyó debido a la concreción de un juicio político que la apartó de su mandato constitucional. Pasada la experiencia petista, para los académicos e investigadores será tiempo de analizar los resultados y legados en las distintas políticas públicas ensayadas en ese lapso de tiempo. En esa dirección, el presente trabajo analizó, a partir del concepto State-Market Condominium, el vínculo entre los gobiernos del PT (20032016) con un actor no gubernamental clave del gigante sudamericano como lo es el empresariado internacionalizado.

Los resultados alcanzados señalan que la relación analizada tuvo tres etapas marcadas en torno a la noción de Condominio. La primera fue la conformación y auge del Condominio (2003-2010) y coincide con los dos gobiernos de Lula Da Silva que desde un principio intentó, a pesar de lo que se esperaba, conciliar postura con las empresas más importantes de Brasil. El mantenimiento de una estabilidad macroeconómica en una etapa de crecimiento de la actividad económica, y el activismo en materia de política exterior -centrado en la denominado "Diplomacia Presidencial”- como instrumento para potenciar la internacionalización del capital brasileño, fueron las variables explicativas de la fortaleza de dicha relación. El segundo momento, que coincide con el primer gobierno de Dilma Rousseff (2011-2014). lo hemos identificado como una retracción relativa del Condominio dado la erosión de los pilares que lo habían permitido. Dicho en otras palabras, las variables explicativas fueron las mismas, pero si en el período anterior funcionaron como elementos positivos para la conformación del Condominio, en esta etapa se transformaron en condicionante negativos. El tercer momento aquí analizado está relacionado con el breve segundo mandato de Dilma Rousseff como Presidenta de Brasil. En un contexto de extrema debilidad política y economía del gobierno del PT se produce la eclosión del Condominio y la emergencia de una situación de State-Market Dichotomydonde los intereses no son solapados sino antagónicos. En esta coyuntura, las variables que explican los cambios deben buscarse alagravamiento de la crisis económica/política y la centralidad la temática “corrupción” en la vida pública de Brasil.

Para finalizar es menester señalar, siguiendo la conceptualización de Underhill, que todo condominio entre un Estado con los actores del mercado se conforma en momentos históricos y con actores económicos particulares, pudiendo en todos los casos desvanecerse en ciertas circunstancias y contextos. Los momentos de tensiones entre el capital financiero internacional y los países desarrollados con la crisis global de 2008 representan un claro ejemplo de la dificultad de conciliar permanentemente intereses solapados entre los distintos estados y los actores del mercado. Motivo por el cual, la evolución del contexto económico y político de Brasil en los próximos años posibilitará, en relación a las grandes empresas internacionalizadas de Brasil, un posible reencauce y restablecimiento del Condominio o, de lo 
contrario, un escenario signado por el antagonismo y la dicotomía y la disputa de intereses.

\section{Agradecimientos}

Este artículo es una versión actualizada de un apartado de la Tesis Doctoral del autor, titulada "Los condicionamientos domésticos en los diseños de política exterior: la internacionalización del capital brasileño y su impacto en la política exterior de Brasil (2003-2013). Implicancias en la relación bilateral con la Argentina”, aprobada en febrero del 2015 (nota 10 Diez-) en el marco del Doctorado en Relaciones Internacionales de la Facultad de Ciencia Política y Relaciones Internacionales de la Universidad Nacional de Rosario, Argentina. 


\section{Notas}

${ }^{1}$ En la literatura especializada es común encontrar como sinónimos los términos trasnacionalización, multinacionalización e internacionalización para describir la expansión global de los capitales. Sin embargo, cada uno de los términos enfatizan distintos aspectos del fenómeno. Mientras que los dos primeros resaltan que una empresa puede tener inversiones en varias naciones y que los accionistas de la misma pueden ser de varias nacionalidades, la idea de internacionalización puntualiza el carácter nacional de los capitales, dado que para internacionalizarse primero necesita ser "nacional". En ese sentido compartimos con el economista brasileño BresserPereira (2010) que: “La enorme mayoría de las 'multinacionales' son corporaciones nacionales pues están controladas por el capital y el conocimiento de uno o dos países [...] estas corporaciones son multinacionales porque tienen presencia en muchos países y no porque su propiedad esté dividida entre un número de países tan grande que las lleva a perder su origen nacional” (p.32)

${ }^{2}$ La democracia brasileña se ha caracterizado por un modelo de "presidencialismo de coalición”. Desde 2004-2013 la coalición de gobierno ha estado formada por el Partido de los Trabajadores (PT) y otros partidos políticos, siendo el Partido del Movimiento Democrático Brasileño (PMDB) el más significativo

${ }^{3}$ El Partido de los Trabajadores fue fundado el 10 de febrero de 1980 . Nació en un contexto de intensas movilizaciones sociales durante la segunda mitad de la década del setenta. Su composición fue heterogénea pero con un fuerte componente de izquierda. Obreros, campesinos, sindicalistas, intelectuales, artistas, entre otros, conformaron el núcleo del partido. Fue el sindicalismo el sector que mayor capacidad de movilización y organización tuvo, producto de su lucha en los años setenta en las famosas huelgas del ABCD paulista. En 1982 fue reconocido como partido político por el Tribunal Superior de Justicia Electoral, formando parte desde entonces del sistema de partidos brasileño.

${ }^{4}$ Dos de los casos más emblemáticos de partidos socialdemócratas europeos que durante las años setenta promulgaban grandes cambios en la estructura económica, y luego en el poder tuvieron políticas pro mercado son el Partido Socialista Español (PSOE) bajo la administración de Felipe González (1982-1996) y el Partido Socialista Francés (PS) bajo la presidencia de François Mitterrand (1981-1995)

${ }^{5}$ La identificación de intereses compartidos tanto por parte del gobierno de Lula como por el empresariado internacionalizado queda claro en los innumerables discursos y declaraciones que realizó el propio Presidente (como sus principales ministros) como también los realizados por actores del sector privado. Para una visualización de los mismos véase Actis (2016)

${ }^{6}$ Discurso del Presidente Lula en la ceremonia de entrega del premio "Woodrow Wilson for Public Service”, el día 21 de septiembre de 2009, Nueva York.

${ }^{7}$ Hacia fines del 2013 el polémico tema del financiamiento privado a las campañas electorales llegó al Supremo Tribunal Federal (STF). Muchos de los Ministros se han manifestado en contra de este tipo de operaciones debido a que varias de las empresas tienen contratos con el Estado. Según Ricardo Lewandowski: "El financiamiento de los partidos y campañas electorales realizadas por empresas son un profundo desequi- 
librio de las luchas electorales, las cuales en democracia deben regirse por el principio de un hombre un voto. A cada ciudadano le debe corresponder un voto, con igual peso e idéntico valor. Las donaciones millonarias hechas por empresas a políticos claramente desfiguran ese principio multisecular" (O Globo, 2014). Recién en septiembre del 2015, luego de la centralidad que tomó el tema de la corrupción entre el empresariado y la clase política brasileña, el STF prohibió que las personas jurídicas puedan aportar a los partidos políticos en las campañas electorales.

${ }^{8}$ La conformación de un condominio no implica la desaparición de contrapuntos y divergencias entre los actores que la integran, máxime cuando se trata de defender posturas corporativas. Por ejemplo, tanto desde la Confederación Nacional de Industria (CNI) como desde la poderosa Federación de Empresarios del Estado de San Pablo (FIESP) han reclamado y hecho público ciertos malestares del empresariado en relación a políticas puntuales llevadas adelante por los gobiernos petistas

${ }^{9}$ Entrevista realizada por el Blog del Planalto al presidente de la empresa Vale, el 7/7/ 2010 en Mozambique. [En línea] http://blog.planalto.gov.br/projeto-da-vale-e-atracaona-tanzania/

${ }^{10}$ Entrevista publicado por el Diario Valor Económico el día 6/12/2007, [En línea] http://www.itamaraty.gov.br/sala-de-imprensa/discursos-artigos-entrevistas-e-outras-

${ }^{11}$ Discurso del Presidente da la República, Luiz Inácio Lula da Silva, por ocasión de la ceremonia de anuncio de inversiones de Embraer en Portugal, 26/7/2008, Lisboa.

12 Variación anual del PBI de Brasil según datos del Banco Mundial: 4,0\% (2006), 6,1\% (2007), 5,2 (2008), -0,3 (2009), 7,5\% (2010), 2,7\% (2011), 1,0\% (2012), 2,5\% (2013).

${ }^{13}$ Para finales del 2012, se comienza acelerar la suba de precio. En 2013 la inflación (5,91\%) superó las metas de inflación propuesta (4,5\%). Por su parte, Brasil comienza a experimentar importante desajustes fiscales (5,8 \% del PBI) y en la balanza de pagos (4,17\% del PBI) en 2014.

${ }^{14}$ Los viajes de Rousseff en comparación con los tres primeros años de los dos mandatos de Lula sustentan lo dicho. De acuerdo a un relevamiento de la BBC (2014), Dilma viajó en ese período de tiempo a 31 países (113 días en el exterior) en comparación con los 49 países (182 días) visitados por Lula durante su primer mandato y los 59 países (288 días) del segundo período

${ }^{15}$ Como bien relata el propio ex Canciller Celso Amorim (2014), su estrecho vínculo de confianza con el Presidente Lula Da Silva fue un elemento central en la activa y coordinada diplomacia que llevó a la práctica Brasil entre 2003 y 2010.

${ }^{16}$ En esa dirección, los dichos de un empresario brasileño en función de las complicaciones de cobrar deudas en Venezuela son significativas “Antes estaba Chávez que era amigo de Lula. Cuando ellos se encontraban destrababan los pagos. Ahora las cosas se complican”. La frase hace una clara alusión a la pérdida del canal político-diplomático privilegiado en el vínculo con Venezuela (Fausto, 2014)

${ }^{17}$ En el año 2002, el grupo empresario argentino PeCom había sido adquirido por 
Petrobras. Entre los activos se encontraba la empresa de distribución eléctrica Transener. Esta situación derivó en preocupaciones por parte del gobierno argentino por la extranjerización de una empresa estratégica. Ante dicha situación el gobierno de Lula se comprometió a que Petrobras vendiera dicho activo nuevamente a la Argentina (aspecto que se concretó recién en 2007). Si bien el margen de acción del gobierno era amplio al ser Petrobras una firma donde la voz del ejecutivo tiene peso, el hecho mostró el interés del gobierno de Lula por ponderar aspectos políticos en cuestiones sensibles para la Argentina.

${ }^{18}$ Discurso de Jorge Gerdau en la 27 $7^{\text {a }}$ edición del "Fórum da Liberdade”, organizado por el Instituto de Estudos Empresariais (IEE Porto Alegre, 8/4/2014)

19 “Abilio Diniz elogia os 3 candidatos, mas vota em Dilma”, 27/8/2014, Exame.com. Disponible en http://exame.abril.com.br/brasil/abilio-diniz-elogia-os-3-candidatos-masvota-em-dilma/

${ }^{20}$ En junio del 2015 el funcionario venezolano Diosdado Cabello visitó Brasil. Además de compromisos oficiales, Cabello se reunió con Joesley Batista dueño de JBS. La firma que Tiene un contrato por US\$2,100 millones y abastece casi la mitad de la carne de res y una cuarta parte de los pollos consumidos por 28 millones de venezolanos carnívoros, consiguió profundizar sus negocios al . Al tomar a su cargo el empaque y la distribución de sus productos en Venezuela. Según Miguel Gularte, presidente de JBS Mercosur "Para JBS, representó la posibilidad de hacer algo que nadie hizo hasta ahora en un país que cuenta con una importante demanda potencial”. Declaraciones realizadas a la agencia Bloomberg 7/9/2015 [En línea] https://www.bloomberg.com/news/ articles/2015-09-07/in-hungry-venezuela-a-brazil-beef-giant-has-extraordinary-power

${ }^{21}$ Discurso pronunciado por Jorge Gerdau Johannpeter el día 7/4/2015 em la Associação Brasileira da Indústria de Máquinas e Equipamentos (Abimaq), [En línea] http:// www. abimaq.org.br/site.as px/Imprens a-Clipping-Tendencias detalhe?DetalheClipping=1224

22 En el marco de la visita oficial del Primer Ministro de la República Popular China a Brasilia, la empresa Vale firmó Memorandos de Entendimiento con el Banco Comercial e Industrial de China (ICBC, siglas en inglés), con el Banco de ImportaciónExportación de China (EXIM Bank) y con dos empresas líderes en transportes del país asiático. Véase [En línea] http://www.itamaraty.gov.br/pt-BR/notas-a-imprensa/9687visita-oficial-do-primeiro-ministro-da-republica-popular-da-china-li-keqiang-ao-brasildocumentos-brasilia-19-de-maio-de-2015

${ }^{23}$ Entrevista realizada a Murilo Ferreira por la cadena Globonews, 18/12/2015 [En línea] http://g1.globo.com/globo-news/noticia/2015/12/presidente-da-vale-prevemelhora-significativa-do-rio-doce-em-10-anos.html

${ }^{24}$ Dado que la principal prueba de las investigaciones realizadas por la justicia brasileña se han basado en el régimen legal del arrepentido (delación premiada) todo el sistema se vio salpicado por las acusaciones cruzadas de los imputados.

${ }^{25}$ En marzo de 2016 el ex presidente Lula fue llevado a declarar por la imputación de una compra de un departamento São Bernardo do Campo a partir de una "propina” de la Constructora OAS así como también de ejercer tráfico de influencia para que muchas 
de las empresas imputadas logren adjudicarse obras en el extranjero. Por su parte, las investigaciones apuntaron a Luciano Coutinho (ex presidente del BNDES) y a la propia Institución por 25 contratos ejecutados por Odebrecht, Andrade Gutierrez, OAS, Queiroz Galvão e Camargo Correa en distintos países de África y Centroamérica. Cabe señalar que la política de financiamiento vía el BNDES fue una de las claves en la profundización del proceso de internacionalización de capitales de Brasil en la primera década de siglo XXI (Sennes y Camargo, 2009) 


\section{Bibliografía}

Actis, E. (2012).Los condicionantes domésticos en los diseños de política exterior: la internacionalización de capitales brasileños como nuevo objetivo de la política exterior de Brasil, Brazilian Journal of International Relations, 2, (3) 39-63.

Actis, E. (2015).Estrategias de Desarrollo e Internacionalización de Capitales: pasado y presente del caso brasileño. Revista Tempo do Mundo, Instituto de Pesquisa Econômica Aplicada (IPEA) .1, (2) 45-68

Actis, E. (2016).Qué fue primero, el huevo o la gallina? Política exterior e internacionalización de capitales en Brasil bajo los gobiernos de Lula Da Silva. Revista POSTdata, 21, (2). 393-421.

Amorim, C. (2014).Breves narrativas diplomáticas. Buenos Aires, Argentina: Taeda

Ban, C. (2013).Brazil's liberal neo-developmentalism: New paradigm or edited orthodoxy?.Review of International Political Economy, 20, (2) 298-331.

Barbosa. N y Pereira De Souza, J. (2010). La inflexión del gobierno de Lula: política económica, crecimiento y distribución. En García, M.A y E. Sader (eds) Brasil entre el pasado y el futuro. Buenos Aires, Argentina: Capital Intelectual.

Bresser-Pereira, L. C. (2010).Globalización y competencia: apuntes para una macroeconomía estructuralista del desarrollo. Buenos Aires, Argentina: Siglo XXI Editora.

Cason, J. y Power, T. (2009).Presidentialization, Pluralization, and the Rollback of Itamaraty: Explaining Change in Brazilian Foreign Policy in the Cardoso-Lula Era. International Political Science Review,30 (2), 117-140.

Castellani, A. (2009) Estado y grandes empresarios en la Argentina de la postconvertibilidad. Revista Cuestione de Sociología, (4-5), 223-234.

Cervo, A. y Lessa, C. (2014). O declínio: inserção internacional do Brasil (2011-2014).Rev. Bras. Polít. Int, 57 (2), 133-151.

Chang, H.J. (2002).Kicking away the Ladder. Development Strategy in historical perspective. London, UK:Wimbledon Publishing Company

Fausto, S. (2014).Não bastam remendos na política externa, Estadão, 19/3/ 2014, [En línea] http://m.estadao.com.br/noticias/opiniao,naobastam-remendos-na-politica-externa-,1142511,0.htm 
Garcia, A. (2011).Políticas públicas e interesses privados: a internacionalização de empresas brasileiras e a atuação internacional do governo Lula, III Encontro Nacional ABRI -Associação Brasileira De Relações Internacionais, 20, 21, 22 de julho, São Paulo.[En línea] http://www.proceedings.scielo.br/scielo.php?pid= MSC0000000122011000100015\&script=sci_arttext

Létourneau, J. (1997).Mundialización e identidades históricas de las regiones.Revista Ciclos, 7 (12) 43-57.

Malamud, A. (2011).La política externa de Dilma Rousseff: ¿menos de lo mismo?Iberoamérica América Latina-España-Portugal. 11 (42)174182.

Moreira, C., Raus, D. y Gómez Leyton, J. C. (2008). La nueva política en América Latina. Rupturas y Continuidades.EnMoreira, C., Raus, D., y Gómez Leyton, J. C. (eds.)La nueva política en América Latina. Ruptura y Continuidades, 7-22. Montevideo, Uruguay: Ediciones Trilce.

Motta Veiga, P. y Polónia Rios, S. (2010).A política externa brasileira sob Lula: o fim do "Consenso de Brasília”, CEBRI artigos, 3 (5), 1-20.

Peralta, P. (2016).Corrupción, electores y política.Revista Nueva Sociedad (Edición Digital). Diciembre. Recuperado de http://nuso.org/articulo/corrupcion-electores-y-politica/

Pereyra Doval. G y Actis. E (2016). The Political and Economic Instability of Dilma Rousseff's Second Government in Brazil: Between Impeachment and the Pragmatic Turn.India Quarterly: A Journal of International Affairs, 72 (2) 120-131

Ribeiro. P, Vizoná. A y Cassotta, P. (2016). Brasil: Un país en compás de espera. Revista de Ciencia Política 36 (1), 51-74

Sennes, R y Camargo R. M. (2009). Public policies and Brazilian multinationals: the rise of Brazilian multinationals. In: Ramsey. J; Almeida, A. (Orgs.).Making the leap from regional heavyweights to true multinationals. Rio de Janeiro, Brasil: Elsevier,

Souza Ramos, L. (2012).Contra hegemonia e política externa. A política externa brasileira no governo Lula. Revista Carta Internacional,7 (1) 69-86.

Stuenkel, O. (2014).O risco do recue estratégico brasileiro.Jornal Folha do S. Paulo. Recuperado de http://www1.folha.uol.com.br/opiniao/2014/ 03/1422485-oliver-stuenkel-o-risco-do-recuo-estrategicobrasileiro.shtml 
Underhill, G. (2003).States, markets and governance for emerging market economies: private interests, the public good and the legitimacy of the development process. International Affairs. (79) 663-689

Valdez, R. C. (julho de 2011). A Atuação do BNDES como Agente Indutor da Inserção Comercial do Brasil no Governo Lula. Em III Econtro Nacional ABRI - Associação Brasileira De Relações Internacionais. São Pablo, Brasil.

Vasconcellos, P. (2014).As Construtoras Brasileiras e o Processo de Integração Regional na América do Sul, Polis Revista Latinoamericana, (39) 1-19. Recuperado de https://polis.revues.org/10461

Vigna, A (2013). Odebrecht, uma transnacional alimentada pelo Estado.Le Monde Diplomatique Brasil. Recuperado de: http:// www.diplomatique.org.br/artigo.php?id=1501

Zibechi, R. (2012).Brasil potencia: entre la integración regional y el nuevo imperialismo. Bogotá, Colombia: Ediciones desde Abajo.

\section{Notas periodísticas}

BBC (14 de fevereiro de 2014) Dilma reduz pela metade viagens internacionais, BBC Brasil. Recuperado de: http://www.bbc.co.uk/ portuguese/noticias/2014/02/140212_viagens_dilma_ms.shtml

Estadão. (27 de fevereiro de 2015). Indonésia ameaça cancelar compra de aviões da Embraer em represália a Dilma. Recuperado de: http:// economia.estadao.com.br/noticias/geral,indonesia-ameaca-cancelarcompra-de-avioes-da-embraer-em-represalia-a-dilma,1638793

Folha Do S.Paulo. (13 de março de 2014). Fazenda diz que pode rediscutir a tributação das multinacionais. Recuperado de: http:// www1.folha.uol.com.br/fsp/mercado/156153-fazenda-diz-que-poderediscutir-a-tributacao-das-multinacionais.shtml

Folha Do S.Paulo (2 de dezembro de 2014b). Indicação de Kátia Abreu para a Agricultura gera atrito com grupo JBS. Recuperado de: http:// www1.folha.uol.com.br/poder/2014/12/1556249-troca-de-ministrogera-atrito-com-grupo-jbs.shtml

La Nación (21 de maio de 2013). Marco Aurelio García: La declaración de Vale nos parece un gran error. Recuperado de:http:// www.lanacion.com.ar/1583802-marco-aurelio-garcia-la-declaracionde-vale-nos-parece-un-gran-error

O Globo (2 de abril de 2014). Maioria do STF vota contra empresa doar a político; decisão final é adiada. Recuperado de:http://g1.globo.com/ 
politica/noticia/2014/04/maioria-do-stf-vota-contra-empresa-doarpolitico-decisao-final-e-adiada.html

Portal Mercosur/ABC (19 de novembro de 2009).Lula da Silva y Cristina Fernández acordaron agenda de negociación. Recuperado de: www.mercosurabc.com.ar/nota.asp?IdNota=2216\&IdSeccion=3

Valor Econômico (25 de abril de 2013a). Vale não levará adiante o projeto de Rio Colorado, diz Murilo Ferreira. Recuperado de: http:// www.valor.com.br/empresas/3101040/vale-nao-levara-adiante-oprojeto-de-rio-colorado-diz-murilo-ferreira

Valor Econômico(26 de abril de 2013b).Após encontro com Cristina, Dilma diz que Vale pode retomar projeto de potássio”. Recuperado de: http://www.valor.com.br/brasil/3101822/apos-encontro-com-cristinadilma-diz-que-vale-pode-retomar-projeto-de-potassio\#ixzz3FNCs6n

Valor Econômico (8 de abril de 2014). Disputa entre BRF e governo. Recuperado de: http://www.valor.com.br/agro/3509060/disputa-entre-brfe-governo

Valor Econômico (23 de fevereiro de 2016). Lucro da Marcopolo cai mais de 60\% em 2015. Recuperado de http://www.valor.com.br/empresas/ 4450054/lucro-da-marcopolo-cai-mais-de-60-em-2015

Recibido: 27.07 .15

Aceptado: 06.02.17 